\title{
A RETINAL GRATICULE
}

BY

\section{O. Gayer Morgan}

LONDON

THIS instrument has been devised to overcome two difficulties which have arisen in the past. The graticule consists of finely ruled millimetre squares subdivided into 0.2 millimetre squares. It is used in conjunction with the Keeler ophthalmoscope, and is mounted on a small slide. In order to focus the image of the graticule on the retina, the retinal vessels are first clearly focussed with the ophthalmoscope, and then the focussing lens is moved up or down until the lines of the squares are seen in perfect definition.

The first and most important difficulty occurs in cases of circumscribed elevations in the fundus often at the macula, and in cases of flat pigmented areas, in both of which it is of great importance to be able to say definitely whether a change in size has taken place over a certain period of time. The diagnosis of malignancy often depends entirely on this fact, as clinically a melanoma, a deep haemorrhage, or exudate is often indistinguishable from a flat sarcoma. At present there is no accurate method except that of making a careful fundus drawing, and as a rule taking a rough comparison with the disc or the distance of the edges of the patch from the main vessels. Neither of these methods gives an accurate measurement for future reference.

The second difficulty has occurred in all descriptions of fundus lesions in publications, where no drawing is added. The retinal graticule as will be explained gives an accurate measurement in millimetres of the size of the part under consideration, and consequently this can be visualized more easily ; also when making a drawing the parts can be accurately measured and reproduced in correct proportions.

The lines of the graticule are most clearly seen in the macular region, but can in most cases be easily counted in any part of the fundus except on the disc. Presumably because there is no definite plane on which they can be focussed, the lines are.very indistinct on the disc margin where the nerve fibres pass over to the retina. This is more noticeable still in papilloedema where the graticule cannot be focussed at all. If necessary a fairly accurate estimate of the transverse measurement of the disc in these cases can be made from the graticule which is clearly in focus above and below. The lines can be clearly seen on the bottom of a physiological or pathological cup, and also on the crescent in 

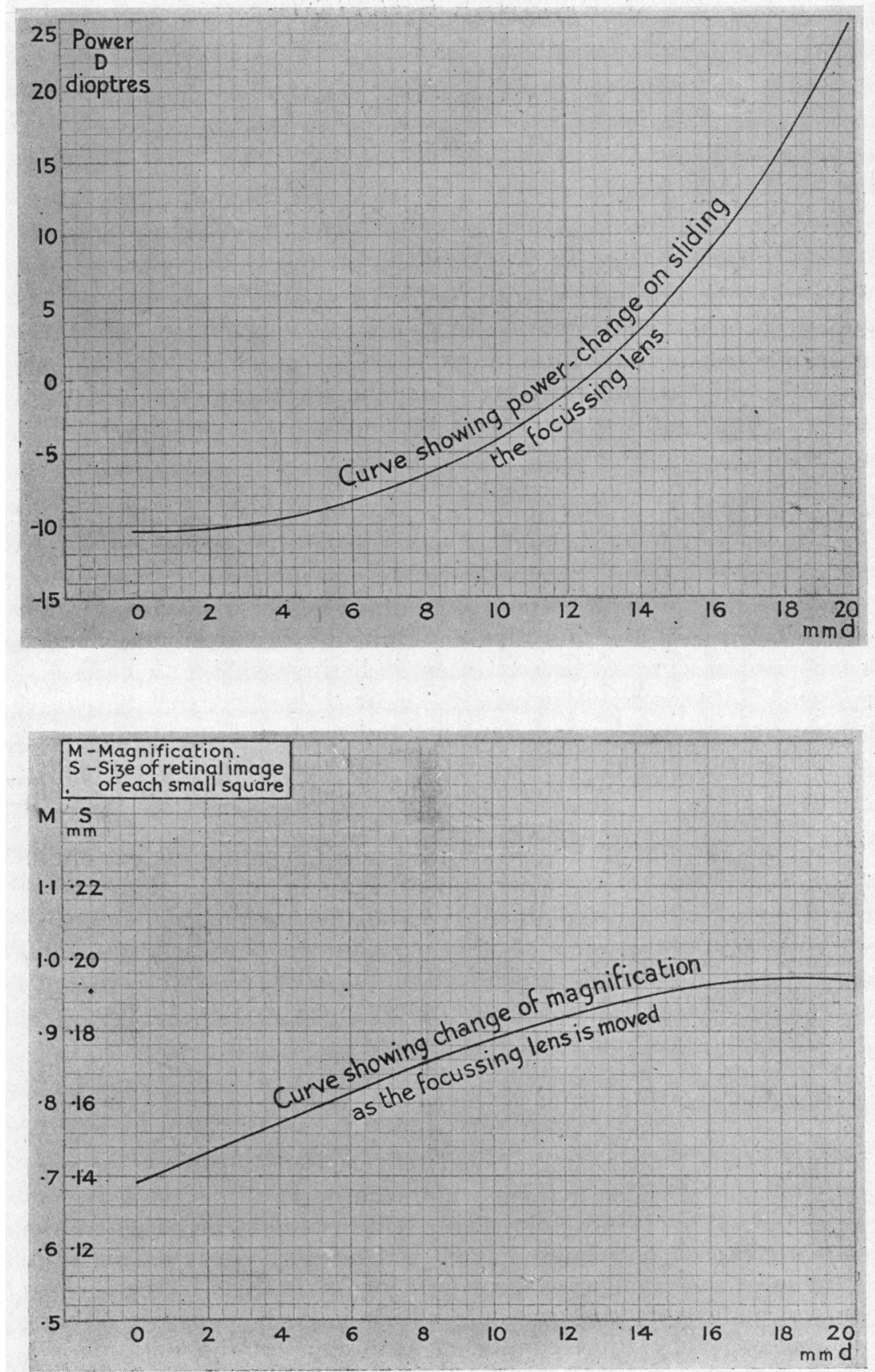
myopic eyes. The transverse measurement of the retinal arteries and veins can be easily estimated and compared.

Magnification.-The magnification produced by the instrument and the size of the retinal image were calculated by the following method. Paraxial rays were traced through the system from an object point at a distance $d$ (variable between 0 and $19.5 \mathrm{~mm}$.). Five values of $d$ were chosen (viz., $0,5,10,15$, and 19.5) to enable curves to be drawn. From these figures the dioptric power of tile light entering the patient's eye was determined as being the reciprocal of the intersection length of the ray leaving the instrument. Also, the magnification of the image on the retina compared with the original graticule size, was calculated by the theorem of Lagrange, that $M=\frac{N U}{N^{1} U^{1}}, N N^{1}$ being the indices of the air and eye respectively; and $U U^{1}$ being the angle of convergence at the graticule and retina respectively.

From the curves the table below is constructed, giving values of the position of the focussing lens, and the ultimate magnification $(M)$ at a number of different refractive powers.

\begin{tabular}{c|c|c}
\hline $\begin{array}{c}\text { Powers in Dioptres. } \\
\text { D. }\end{array}$ & $\begin{array}{c}\text { Shift of focussing } \\
\text { lens. } \\
\text { D.=mm. }\end{array}$ & $\begin{array}{c}\text { Size of each square. } \\
\text { Approximate } \\
\text { Magnification. M. }\end{array}$ \\
\hline 10 & 16.4 & 0.192 \\
8 & 15.7 & 0.192 \\
6 & 15.1 & 0.192 \\
4 & 14.3 & 0.190 \\
3 & 13.9 & 0.190 \\
2 & 13.5 & 0.188 \\
1 & 13.0 & 0.188 \\
0 & 12.5 & 0.186 \\
-1 & 12.0 & 0.182 \\
-2 & 11.4 & 0.180 \\
-3 & 10.8 & 0.178 \\
-4 & 10.2 & 0.172 \\
-8 & 8.5 & 0.164 \\
-10 & 6.2 & 0.150 \\
\hline
\end{tabular}

The graticule is made of $\mathrm{mm}$. squares, subdivided into $0.2 \mathrm{~mm}$. squares. With an emmetropic eye $(\mathrm{D}=\mathrm{O})$, the magnification is $0.93 \mathrm{~mm}$. hence each square covers $0.186 \mathrm{~mm}$. of the actual retina itself. For myopic or hypermetropic eyes, the figures in the table for magnification assume that the error in the refraction is caused solely by elongation or shortening of the eyeball, and not by any error of the crystalline lens; if, however, the latter is the case, then a new table of magnification will be necessary, but the greatest error would not be more than $15-20$ per cent. of those given in the table. 